\title{
Nonlinear systems with a partial Nash type equilibrium
}

\author{
Andrei Stan
}

\begin{abstract}
In this paper fixed point arguments and a critical point technique are combined leading to hybrid existence results for a system of three operator equations where only two of the equations have a variational structure. The components of the solution which are associated to the equations having a variational form represent a Nash-type equilibrium of the corresponding energy functionals. The result is achieved by an iterative scheme based on Ekeland's variational principle.
\end{abstract}

Mathematics Subject Classification (2010): 47H10, 47J30, 34C25.

Keywords: Nash-type equilibrium, Perov contraction, Ekeland variational principle, periodic solution.

\section{Introduction}

Many nonlinear equations can be seen as a problem of fixed point $N(u)=u$, where $N$ is a certain operator. One says that the equation has a variational form if it is equivalent with a critical point equation $E^{\prime}(u)=0$. In the paper [7], R. Precup studied systems of the form

$$
\left\{\begin{array}{l}
N_{1}(u, v)=u \\
N_{2}(u, v)=v
\end{array}\right.
$$

in a Hilbert space, where each of the equations has a variational form, i.e. there are two $C^{1}$ functionals $E_{1}$ and $E_{2}$ such that

$$
\begin{aligned}
& E_{11}(u, v)=u-N_{1}(u, v) \text { and } \\
& E_{22}(u, v)=v-N_{2}(u, v),
\end{aligned}
$$

where $E_{11}$ and $E_{22}$ are the partial Fréchet derivatives of $E_{1}$ and $E_{2}$ with respect to $\mathrm{u}$ and $\mathrm{v}$, respectively. Sufficient conditions have been established for that the system 
admits a solution which is a Nash type equilibrium for the functionals $E_{1}$ and $E_{2}$, that is

$$
\begin{aligned}
& E_{1}(u, v)=\inf _{u} E(\cdot, v), \\
& E_{2}(u, v)=\inf _{v} E(u, \cdot) .
\end{aligned}
$$

Related results are obtained in [1].

The concept of a Nash equilibrium goes back to 1838 when Antoine Augustin Cournot [3] used it in his economics studies about the best output of a firm depending on the outputs of the other firms. The existence of such an equilibrium in the framework of the game theory was proved later in 1951 by John Forbes Nash Jr [5] by using Brouwer's fixed point theorem. Now the concept is also used outside economics to systems of variational equations. From a physical point of view, a Nash-type equilibrium $(u, v)$ for two interconnected mechanisms whose energies are $E_{1}, E_{2}$ is such that the motion of each mechanism is conformed to the minimum energy principle by taking into account the motion of the other.

Also, in the paper [2], a system of type (1.1) is studied under the assumption that only one of the equations, say the second one, has a variational form, and the authors prove the existence of a solution $(u, v)$ such that $v$ minimizes $E(u, \cdot)$, where $E$ is the energy functional associated with the second equation. For the proof, they use a hybrid fixed point - critical point method based on Banach's contraction theorem and Ekeland's variational principle.

The aim of this paper is to combine the techniques used in [7] and [2], for the study of a system of three equations

$$
\left\{\begin{array}{l}
N_{1}(u, v, w)=u \\
N_{2}(u, v, w)=v \\
N_{3}(u, v, w)=w
\end{array}\right.
$$

where only the last two equations have a variational form. Our goal is to obtain a solution $(u, v, w)$ such that the pair $(v, w)$ is a Nash type equilibrium for the two functionals associated to the last two equations.

\section{Main result}

Let $\left(X_{1}, d\right)$ be a complete metric space and $\left(X_{2},|\cdot|_{2}\right),\left(X_{3},|\cdot|_{3}\right)$ be two real Hilbert spaces which are identified with their duals. Denote $X:=X_{1} \times X_{2} \times X_{3}$. Let $N_{i}: X \rightarrow X_{i}(i=1,2,3)$ be continuous and assume that $N_{2}, N_{3}$ have a variational structure, i.e. there exist functionals $E_{2}, E_{3}: X \rightarrow \mathbb{R}$ such that $E_{2}(u, \cdot, w)$ is Fréchet differentiable for every $(u, w) \in X_{1} \times X_{3}, E_{3}(u, v, \cdot)$ is Fréchet differentiable for every $(u, v) \in X_{1} \times X_{2}$ and

$$
\begin{aligned}
& E_{22}(u, v, w)=v-N_{2}(u, v, w), \\
& E_{33}(u, v, w)=w-N_{3}(u, v, w) .
\end{aligned}
$$

Here $E_{22}, E_{33}$ are the Fréchet derivatives of $E_{2}(u, \cdot, w)$ and $E_{3}(u, v, \cdot)$, respectively. 
We also assume that the operator $N: X \rightarrow X$,

$$
N(u, v, w)=\left(N_{1}(u, v, w), N_{2}(u, v, w), N_{3}(u, v, w)\right)
$$

is a Perov contraction, i.e. there is a square matrix $A=\left[a_{i j}\right]_{1 \leq i, j \leq 3} \in \mathbf{M}_{3}\left(\mathbb{R}_{+}\right)$such that $A^{k}$ tends to the zero matrix $0_{3}$ as $k \rightarrow \infty$ and the following vector Lipschitz condition is satisfied

$$
\left[\begin{array}{l}
d\left(N_{1}(u, v, w), N_{1}(\bar{u}, \bar{v}, \bar{w})\right) \\
\left|N_{2}(u, v, w)-N_{2}(\bar{u}, \bar{v}, \bar{w})\right|_{2} \\
\left|N_{3}(u, v, w)-N_{3}(\bar{u}, \bar{v}, \bar{w})\right|_{3}
\end{array}\right] \leq A\left[\begin{array}{c}
d(u, \bar{u}) \\
|v-\bar{v}|_{2} \\
|w-\bar{w}|_{3}
\end{array}\right]
$$

for every $(u, v, w) \in X$.

Note that the for a square matrix $A \in \mathbf{M}_{n}\left(\mathbb{R}_{+}\right)$, condition $A^{k}$ tends to the zero matrix $0_{n}$ as $k \rightarrow \infty$ is equivalent (see [6]) to each one of the following properties:

(i) The spectral radius of $A$ is less than one;

(ii) $I_{n}-A$ is invertible and $\left(I_{n}-A\right)^{-1} \in \mathbf{M}_{n}\left(\mathbb{R}_{+}\right)$;

(iii) $I_{n}-A$ is invertible and $I_{n}+M+M^{2}+\ldots=\left(I_{n}-A\right)^{-1}$.

Here $I_{n}$ stands for the unit matrix in $\mathbf{M}_{n}(\mathbb{R})$.

The main result is the following theorem.

Theorem 2.1. Assume that the above conditions are satisfied. Moreover assume that $E_{2}(u, \cdot, w), E_{3}(u, v, \cdot)$ are bounded from below for every $(u, v, w) \in X$ and that are constants $R_{2}, R_{3}, a>0$ such that

$$
\begin{aligned}
& E_{2}(u, v, w) \geq \inf _{X_{2}} E_{2}(u, \cdot, w)+a \quad \text { for all }(u, w) \in X_{1} \times X_{3} \text { and }|v|_{2} \geq R_{2}, \\
& E_{3}(u, v, w) \geq \inf _{X_{3}} E_{3}(u, v, \cdot)+a \quad \text { for all }(u, v) \in X_{1} \times X_{2} \text { and }|w|_{3} \geq R_{3} .
\end{aligned}
$$

Then the unique fixed point $\left(u^{*}, v^{*}, w^{*}\right)$ ensured by the Perov contraction theorem has the property that $\left(v^{*}, w^{*}\right)$ is a Nash type equilibrium for the pair of functionals $\left(E_{2}, E_{3}\right)$, i.e.

$$
\begin{aligned}
& E_{2}\left(u^{*}, v^{*}, w^{*}\right)=\inf _{X_{2}} E_{2}\left(u^{*}, \cdot, w^{*}\right), \\
& E_{3}\left(u^{*}, v^{*}, w^{*}\right)=\inf _{X_{3}} E_{3}\left(u^{*}, v^{*}, \cdot\right) .
\end{aligned}
$$

For the proof we need alternatively one of the following two auxiliary results.

Lemma 2.2. Let $\left(A_{k, p}\right)_{k \geq 1},\left(B_{k, p}\right)_{k \geq 1}$ be two sequences of vectors in $\mathbb{R}_{+}^{n}$ (column vectors) depending on a parameter $p$, such that

$$
A_{k, p} \leq M A_{k-1, p}+B_{k, p}
$$

for all $k$ and $p$, where $M \in \mathbf{M}_{n}\left(\mathbb{R}_{+}\right)$is a matrix with spectral radius less than one. If the sequence $\left(A_{k, p}\right)_{k \geq 1}$ is bounded uniformly with respect to $p$ and $B_{k, p} \rightarrow 0_{n}$ as $k \rightarrow \infty$ uniformly with respect to $p$, then $A_{k, p} \rightarrow 0_{n}$ as $k \rightarrow \infty$ uniformly with respect to $p$. 
Proof. Since $B_{k, p} \rightarrow 0_{n}$ as $k \rightarrow \infty$ uniformly with respect to $p$, for any fixed column vector $\epsilon \in(0, \infty)^{n}$, we can find $k_{1}$ independent of $p$ such that $B_{k, p} \leq \epsilon$ for all $k \geq k_{1}$ and all $p$. Then, for $k>k_{1}$ we have

$$
\begin{aligned}
A_{k, p} & \leq M A_{k-1, p}+\epsilon \leq M^{2} A_{k-2, p}+\epsilon+M \epsilon \\
& \leq \ldots \\
& \leq M^{k-k_{1}} A_{k_{1}, p}+\epsilon\left(I_{n}+M+\ldots M^{k-k_{1}}\right) \\
& \leq M^{k-k_{1}} A_{k_{1}, p}+\epsilon\left(I_{n}-M\right)^{-1} .
\end{aligned}
$$

The conclusion now follows since $\left(A_{k, p}\right)_{k \geq 1}$ is bounded uniformly with respect to $p$ and $M^{k-k_{1}} \rightarrow 0_{n}$ as $k \rightarrow \infty$.

Lemma 2.3. Let $\left(x_{k, p}\right)_{k \geq 1},\left(y_{k, p}\right)_{k \geq 1}$ be two sequences of nonnegative real numbers depending on a parameter $p$ which are bounded uniformly with respect to $p$. Assume that for all $k$ and $p$,

$$
a x_{k, p}+b y_{k, p} \leq a^{\prime} x_{k-1, p}+b^{\prime} y_{k-1, p}+q_{k, p}
$$

where $0<a^{\prime}<a, 0 \leq b^{\prime}<b, \frac{b^{\prime}}{a^{\prime}}<\frac{b}{a}$, and $\left(q_{k, p}\right)_{k \geq 1}$ is a sequence of positive real numbers converging to zero uniformly with respect to $p$. Then $x_{k, p} \rightarrow 0$ and $y_{k, p} \rightarrow 0$ as $k \rightarrow \infty$ uniformly with respect to $p$.

Proof. By the uniform convergence to zero of $q_{k, p}$, taking $\epsilon>0$, we can find $k_{1}$ independent of $p$, such that $\frac{q_{k, p}}{a}<\epsilon$ for all $k>k_{1}$. Consider $k>k_{1}$ and assume $a>b$. Then

$$
\begin{aligned}
x_{k, p}+\frac{b}{a} y_{k, p} & \leq \frac{a^{\prime}}{a}\left(x_{k-1, p}+\frac{b^{\prime}}{a^{\prime}} y_{k-1, p}\right)+\epsilon \leq \frac{a^{\prime}}{a}\left(x_{k-1, p}+\frac{b}{a} y_{k-1, p}\right)+\epsilon \\
& \leq\left(\frac{a^{\prime}}{a}\right)^{2}\left(x_{k-2, p}+\frac{b^{\prime}}{a^{\prime}} y_{k-2, p}\right)+\epsilon\left(\frac{a^{\prime}}{a}+1\right) \\
& \ldots \\
& \leq\left(\frac{a^{\prime}}{a}\right)^{k-k_{1}}\left(x_{k_{1}, p}+\frac{b^{\prime}}{a^{\prime}} y_{k_{1}, p}\right)+\epsilon\left(\left(\frac{a^{\prime}}{a}\right)^{k-k_{1}-1}+\ldots+1\right) \\
& \leq\left(\frac{a^{\prime}}{a}\right)^{k-k_{1}}\left(x_{k_{1}, p}+\frac{b^{\prime}}{a^{\prime}} y_{k_{1}, p}\right)+\epsilon \frac{1}{1-\frac{a^{\prime}}{a}} .
\end{aligned}
$$

Taking into account that $\frac{a^{\prime}}{a}<1$ and the boundedness of $x_{k, p}$ and $y_{k, p}$, it is clear that $x_{k, p}+\frac{b}{a} y_{k, p} \rightarrow 0$ as $k \rightarrow \infty$ uniformly with respect to $p$. This clearly gives the conclusion. The case $a<b$ can be treated analogously.

Proof of the theorem. First note that since the spectral radius of matrix $A$ is less than one, the elements $a_{i i}$ of the main diagonal are less than one. Consequently, for every $(v, w) \in X_{2} \times X_{3}$, the operator $N_{1}(\cdot, v, w): X_{1} \rightarrow X_{1}$ is a contraction. We now use an iterative procedure to construct an approximating sequence $\left(u_{k}, v_{k}, w_{k}\right)$. We start with some fixed element $\left(v_{0}, w_{0}\right) \in X_{2} \times X_{3}$. Then, by Banach contraction principle, 
there exists $u_{1} \in X_{1}$ such that $N_{1}\left(u_{1}, v_{0}, w_{0}\right)=u_{1}$. Next, for fixed $\left(u_{1}, w_{0}\right)$, according to Ekeland variational principle, there is $v_{1} \in X_{2}$ such that

$$
E_{2}\left(u_{1}, v_{1}, w_{0}\right) \leq \inf _{X_{2}} E_{2}\left(u_{1}, \cdot, w_{0}\right)+1, \quad\left|E_{22}\left(u_{1}, v_{1}, w_{0}\right)\right|_{2} \leq 1
$$

Using again Ekeland variational principle for fixed $\left(u_{1}, v_{1}\right)$, there is $w_{1} \in X_{3}$ with

$$
E_{3}\left(u_{1}, v_{1}, w_{1}\right) \leq \inf _{X_{3}} E_{2}\left(u_{1}, v_{1}, \cdot\right)+1, \quad\left|E_{33}\left(u_{1}, v_{1}, w_{1}\right)\right|_{3} \leq 1 .
$$

At step $k$, we find a triple $\left(u_{k}, v_{k}, w_{k}\right)$ having the following proprieties:

$$
\begin{aligned}
& N_{1}\left(u_{k}, v_{k-1}, w_{k-1}\right)=u_{k}, \\
& E_{2}\left(u_{k}, v_{k}, w_{k-1}\right) \leq \inf _{X_{2}} E_{2}\left(u_{k}, \cdot, w_{k-1}\right)+\frac{1}{k},\left|E_{22}\left(u_{k}, v_{k}, w_{k-1}\right)\right|_{2} \leq \frac{1}{k}, \\
& E_{3}\left(u_{k}, v_{k}, w_{k}\right) \leq \inf _{X_{3}} E_{3}\left(u_{k}, v_{k}, \cdot\right)+\frac{1}{k}, \mid E_{33}\left(u_{k}, v_{k},\left.w_{k}\right|_{3} \leq \frac{1}{k} .\right.
\end{aligned}
$$

Our next task is to prove that the sequences $u_{k}, v_{k}, w_{k}$ are Cauchy, which will ensure their convergence. Since $N_{1}\left(u_{k}, v_{k-1}, w_{k-1}\right)=u_{k}$, we have

$$
\begin{aligned}
d\left(u_{k+p}, u_{k}\right) & =d\left(N_{1}\left(u_{k+p}, v_{k+p-1}, w_{k+p-1}\right), N_{1}\left(u_{k}, v_{k-1}, w_{k-1}\right)\right) \\
& \leq a_{11} d\left(u_{k+p}, u_{k}\right)+a_{12}\left|v_{k+p-1}-v_{k-1}\right|_{2}+a_{13}\left|w_{k+p-1}-w_{k-1}\right|_{3},
\end{aligned}
$$

whence

$$
d\left(u_{k+p}, u_{k}\right) \leq \frac{a_{12}}{1-a_{11}}\left|v_{k+p-1}-v_{k-1}\right|_{2}+\frac{a_{13}}{1-a_{11}}\left|w_{k+p-1}-w_{k-1}\right|_{3} .
$$

For the sequence $\left(v_{k}\right)$ and $\left(w_{k}\right)$ we have

$$
\begin{aligned}
&\left|v_{k+p}-v_{k}\right|_{2} \leq\left|-N_{2}\left(u_{k+p}, v_{k+p}, w_{k+p-1}\right)+v_{k+p}-v_{k}+N_{2}\left(u_{k}, v_{k}, w_{k-1}\right)\right|_{2} \\
&+\left|N_{2}\left(u_{k+p}, v_{k+p}, w_{k+p-1}\right)-N_{2}\left(u_{k}, v_{k}, w_{k-1}\right)\right|_{2}, \\
&\left|w_{k+p}-w_{k}\right|_{3} \leq\left|-N_{3}\left(u_{k+p}, v_{k+p}, w_{k+p}\right)+w_{k+p}-w_{k}+N_{3}\left(u_{k}, v_{k}, w_{k}\right)\right|_{3} \\
&+\left|N_{3}\left(u_{k+p}, v_{k+p}, w_{k+p}\right)-N_{3}\left(u_{k}, v_{k}, w_{k}\right)\right|_{3} .
\end{aligned}
$$

Denote

$$
\begin{aligned}
\beta_{k, p} & :=\left|-N_{2}\left(u_{k+p}, v_{k+p}, w_{k+p-1}\right)+v_{k+p}-v_{k}+N_{2}\left(u_{k}, v_{k}, w_{k-1}\right)\right|_{2} \\
= & \left.\mid E_{22}\left(u_{k+p}, v_{k+p}, w_{k+p-1}\right)-E_{22}\left(u_{k}, v_{k}, w_{k-1}\right)\right)\left.\right|_{2}, \\
\gamma_{k, p} & :=\left|-N_{3}\left(u_{k+p}, v_{k+p}, w_{k+p}\right)+w_{k+p}-w_{k}+N_{3}\left(u_{k}, v_{k}, w_{k}\right)\right|_{3} \\
& \left.=\mid E_{33}\left(u_{k+p}, v_{k+p}, w_{k+p}\right)-E_{33}\left(u_{k}, v_{k}, w_{k-}\right)\right)\left.\right|_{3}, \\
x_{k, p} & :=d\left(u_{u+p}, u_{k}\right), \quad y_{k, p}:=\left|v_{k+p}-v_{k}\right|_{2}, \quad z_{k, p}:=\left|w_{k+p}-w_{k}\right|_{3} .
\end{aligned}
$$

With these notations, using (2.5), (2.6) and the Perov contraction condition, we obtain

$$
\begin{aligned}
& x_{k, p} \leq a_{11} x_{k, p}+a_{12} y_{k-1, p}+a_{13} z_{k-1, p}, \\
& y_{k, p} \leq a_{21} x_{k, p}+a_{22} y_{k, p}+a_{23} z_{k-1, p}+\beta_{k, p}, \\
& z_{k, p} \leq a_{31} x_{k, p}+a_{32} y_{k, p}+a_{33} z_{k, p}+\gamma_{k, p} .
\end{aligned}
$$

For the continuation of the proof we may use either Lemma 2.2 or Lemma 2.3. 
1) Use of Lemma 2.2. Letting

$$
A^{\prime}=\left[\begin{array}{ccc}
a_{11} & 0 & 0 \\
a_{21} & a_{22} & 0 \\
a_{31} & a_{32} & a_{33}
\end{array}\right] \text { and } A^{\prime \prime}=A-A^{\prime}
$$

the following inequality holds

$$
\left[\begin{array}{l}
x_{k, p} \\
y_{k, p} \\
z_{k, p}
\end{array}\right] \leq A^{\prime}\left[\begin{array}{l}
x_{k, p} \\
y_{k, p} \\
z_{k, p}
\end{array}\right]+A^{\prime \prime}\left[\begin{array}{l}
x_{k-1, p} \\
y_{k-1, p} \\
z_{k-1, p}
\end{array}\right]+\left[\begin{array}{c}
0 \\
\beta_{k, p} \\
\gamma_{k, p}
\end{array}\right] .
$$

Note that if $\rho(A)<1$, than also $\rho\left(A^{\prime}\right)<1$. Indeed, one clearly has $A^{\prime k}<A^{k}$, and so if $A^{k} \rightarrow 0$ as $k \rightarrow \infty$, then $A^{\prime k} \rightarrow 0$ too.

Rewriting (2.10) as

$$
\left(I-A^{\prime}\right)\left[\begin{array}{l}
x_{k, p} \\
y_{k, p} \\
z_{k, p}
\end{array}\right] \leq A^{\prime \prime}\left[\begin{array}{l}
x_{k-1, p} \\
y_{k-1, p} \\
z_{k-1, p}
\end{array}\right]+\left[\begin{array}{c}
0 \\
\beta_{k, p} \\
\gamma_{k, p}
\end{array}\right]
$$

and using the fact that $I-A^{\prime}$ is invertible and its inverse has positive entries, we can multiply by $\left(I-A^{\prime}\right)^{-1}$ to obtain

$$
\left[\begin{array}{l}
x_{k, p} \\
y_{k, p} \\
z_{k, p}
\end{array}\right] \leq\left(I-A^{\prime}\right)^{-1} A^{\prime \prime}\left[\begin{array}{l}
x_{k-1, p} \\
y_{k-1, p} \\
z_{k-1, p}
\end{array}\right]+\left(I-A^{\prime}\right)^{-1}\left[\begin{array}{c}
0 \\
\beta_{k, p} \\
\gamma_{k, p}
\end{array}\right] .
$$

Observe that $M:=\left(I-A^{\prime}\right)^{-1} A^{\prime \prime}$ has the spectral radius less than one. To prove this, it is enough to show that $I-M$ is invertible with the inverse has nonegative entries. Is clear that

$$
\begin{aligned}
M & =\left(I-A^{\prime}\right)^{-1} A^{\prime \prime}=\left(I-A^{\prime}\right)^{-1}\left(A-A^{\prime}\right)=\left(I-A^{\prime}\right)^{-1}\left(I-A^{\prime}+A-I\right) \\
& =I-\left(I-A^{\prime}\right)^{-1}(I-A),
\end{aligned}
$$

hence $I-M=\left(I-A^{\prime}\right)^{-1}(I-A)$. Because $\left(I-A^{\prime}\right)^{-1}$ and $I-A$ are invertible, by taking $Q:=(I-A)^{-1}\left(I-A^{\prime}\right)$, we have $Q(I-M)=(I-M) Q=I$, hence $I-M$ is invertible and its inverse is $Q$. One has

$$
Q=(I-A)^{-1}\left(I-A^{\prime}\right)=(I-A)^{-1}\left(I-A+A^{\prime \prime}\right)=I+(I-A)^{-1} A^{\prime \prime}
$$

and since $(I-A)^{-1} A^{\prime \prime}$ and $I$ are positive matrices, it follows that $Q$ is also positive. Therefore, the spectral radius of $M$ is less than one.

From (2.2) and (2.3) we have that $y_{k, p}$ and $z_{k, p}$ are bounded uniformly with respect to $p$. Because of this, is immediate that $x_{k, p}$ is also bounded uniformly with respect to $p$. Moreover, it is clear that

$$
\left[\begin{array}{c}
0 \\
\beta_{k, p} \\
\gamma_{k, p}
\end{array}\right]
$$

converges to zero uniformly with respect to $p$. Applying Lemma 2.2 we obtain that $x_{k, p}, y_{k, p}, z_{k, p}$ are convergent to zero uniformly with respect to $p$. Hence the sequences $u_{k}, v_{k}$ and $w_{k}$ are Cauchy as desired. 
2) Use of Lemma 2.3. The relations $(2.7),(2.8),(2.9)$ can be rewritten under the form

$$
\begin{aligned}
& x_{k, p} \leq a_{11} x_{k, p}+a_{12} y_{k, p}+a_{13} z_{k, p}+a_{12}\left(y_{k-1, p}-y_{k, p}\right)+a_{13}\left(z_{k-1, p}-z_{k, p}\right), \\
& y_{k, p} \leq a_{21} x_{k, p}+a_{22} y_{k, p}+a_{23} z_{k, p}+\beta_{k, p}+a_{23}\left(z_{k-1, p}-z_{k, p}\right), \\
& z_{k, p} \leq a_{31} x_{k, p}+a_{32} y_{k, p}+a_{33} z_{k, p}+\gamma_{k, p},
\end{aligned}
$$

which can be put under the vector form

$$
\left[\begin{array}{l}
x_{k, p} \\
y_{k, p} \\
z_{k, p}
\end{array}\right] \leq A\left[\begin{array}{l}
x_{k, p} \\
y_{k, p} \\
z_{k, p}
\end{array}\right]+\left[\begin{array}{c}
a_{12}\left(y_{k-1, p}-y_{k, p}\right)+a_{13}\left(z_{k-1, p}-z_{k, p}\right) \\
\beta_{k, p}+a_{23}\left(z_{k-1, p}-z_{k, p}\right) \\
\gamma_{k, p}
\end{array}\right]
$$

Denoting $(I-A)^{-1}=C=\left[c_{i j}\right]_{1 \leq i, j \leq 3}$ we have

$$
\left[\begin{array}{l}
x_{k, p} \\
y_{k, p} \\
z_{k, p}
\end{array}\right] \leq C\left[\begin{array}{c}
a_{12}\left(y_{k-1, p}-y_{k, p}\right)+a_{13}\left(z_{k-1, p}-z_{k, p}\right) \\
\beta_{k, p}+a_{23}\left(z_{k-1, p}-z_{k, p}\right) \\
\gamma_{k, p}
\end{array}\right],
$$

whence

$$
\begin{aligned}
y_{k, p} & \leq c_{21} a_{12}\left(y_{k-1, p}-y_{k, p}\right)+c_{21} a_{13}\left(z_{k-1, p}-z_{k, p}\right) \\
& +c_{22} a_{23}\left(z_{k-1, p}-z_{k, p}\right)+c_{22} \beta_{k, p}+c_{33} \gamma_{k, p}, \\
z_{k, p} & \leq c_{31} a_{12}\left(y_{k-1, p}-y_{k, p}\right)+c_{31} a_{13}\left(z_{k-1, p}-w_{k, p}\right) \\
& +c_{32} a_{23}\left(z_{k-1, p}-z_{k, p}\right)+c_{32} \beta_{k, p}+c_{33} \gamma_{k, p} .
\end{aligned}
$$

We make the following notations

$$
\begin{aligned}
& a^{\prime}=c_{12} a_{13}+c_{22} a_{2,3}+c_{31} a_{13}+c_{32} a_{23}, \\
& b^{\prime}=c_{21} a_{12}+c_{31} a_{12} .
\end{aligned}
$$

Adding (2.11) and (2.12) we obtain

$y_{k, p}+z_{k, p} \leq a^{\prime} y_{k-1, p}-a^{\prime} y_{k, p}+b^{\prime} z_{k-1, p}-b^{\prime} z_{k, p}+c_{22} \beta_{k, p}+c_{33} \gamma_{k, p}+c_{32} \beta_{k, p}+c_{33} \gamma_{k, p}$, whence, with the notations $a=1+a^{\prime}, b=1+b^{\prime}$ and

$$
q_{k, p}:=c_{22} \beta_{k, p}+c_{33} \gamma_{k, p}+c_{32} \beta_{k, p}+c_{33} \gamma_{k, p},
$$

one has

$$
a y_{k, p}+b z_{k, p} \leq a^{\prime} y_{k-1, p}+b^{\prime} z_{k-1, p}+q_{k, p} .
$$

Note that the sequence $q_{k, p}:=c_{22} \beta_{k, p}+c_{33} \gamma_{k, p}+c_{32} \beta_{k, p}+c_{33} \gamma_{k, p}$ converges to zero as $k \rightarrow \infty$ uniformly with respect to $p$, and that from (2.2) and (2.3), the sequences $\left(y_{k, p}\right)_{k \geq 1},\left(z_{k, p}\right)_{k \geq 1}$ are bounded uniformly with respect to $p$. Also note that if $b^{\prime}<a^{\prime}$, then $\frac{b^{\prime}}{a^{\prime}}<\frac{b}{a}$ and from Lemma 2.3 we obtain that $y_{k, p}$ and $z_{k, p}$ converge to zero as $k \rightarrow \infty$ uniformly with respect to $p$. Similarly, if $a^{\prime}<b^{\prime}$, then we obtain the same conclusion if we apply Lemma 2.3 by interchanging $a$ with $b$ and $y_{k, p}$ with $z_{k, p}$. Next, from (2.7) we deduce that $x_{k, p} \rightarrow 0$ as $k \rightarrow \infty$ uniformly with respect to $p$, and as above, that the sequences $u_{k}, v_{k}$ and $w_{k}$ are Cauchy as desired.

Finally the limits $u^{*}, v^{*}, w^{*}$ of the sequences $u_{k}, v_{k}$ and $w_{k}$ give the desired solution of the system after passing to the limit in (2.4). 


\section{Application}

Consider the system

$$
\left\{\begin{array}{l}
-u^{\prime \prime}+a_{1}^{2} u=f_{1}\left(t, u(t), v(t), w(t), u^{\prime}(t)\right) \\
-v^{\prime \prime}+a_{2}^{2} v=\nabla_{y} f_{2}(t, u(t), v(t), w(t)) \\
-w^{\prime \prime}+a_{3}^{2} w=\nabla_{z} f_{3}(t, u(t), v(t), w(t))
\end{array}\right.
$$

with the periodic conditions

$$
\begin{aligned}
u(0)-u(T) & =u^{\prime}(0)-u^{\prime}(T)=0, \\
v(0)-v(T) & =v^{\prime}(0)-v^{\prime}(T)=0, \\
w(0)-w(T) & =w^{\prime}(0)-w^{\prime}(T)=0,
\end{aligned}
$$

where $f_{2}, f_{3}:(0, T) \times \mathbb{R}^{k_{1}} \times \mathbb{R}^{k_{2}} \times \mathbb{R}^{k_{3}} \rightarrow \mathbb{R}$ and $f_{1}:(0, T) \times \mathbb{R}^{k_{1}} \times \mathbb{R}^{k_{2}} \times \mathbb{R}^{k_{3}} \times \mathbb{R}^{k_{1}} \rightarrow \mathbb{R}^{k_{1}}$. We will assume that $f_{1}, f_{2}, f_{3}, \nabla_{y} f_{2}$ and $\nabla_{z} f_{3}$ are $L^{1}$ - Carathéodory functions.

For $i=1,2,3$, let $H_{p}^{1}\left(0, T ; \mathbb{R}^{k_{i}}\right)$ be the closure in $H^{1}\left(0, T ; \mathbb{R}^{k_{i}}\right)$ of the space $\left\{u \in C^{1}\left([0, T] ; \mathbb{R}^{k_{i}}\right): u(0)=u(T), u^{\prime}(0)=u^{\prime}(T)\right\}$. We shall endow this space with the inner product

$$
(u, v)_{i}:=\left(u^{\prime}, v^{\prime}\right)_{L^{2}\left(0, T ; \mathbb{R}^{k_{i}}\right)}+a_{i}^{2}(u, v)_{L^{2}\left(0, T ; \mathbb{R}^{k_{i}}\right)}
$$

and the corresponding norm

$$
|u|_{i}=\left(\left|u^{\prime}\right|_{L^{2}\left(0, T ; \mathbb{R}^{k_{i}}\right)}^{2}+a_{i}^{2}|u|_{L^{2}\left(0, T ; \mathbb{R}^{k_{i}}\right)}^{2}\right)^{\frac{1}{2}} .
$$

Also we consider the operator $J_{i}:\left(H_{p}^{1}\left(0, T ; \mathbb{R}^{k_{i}}\right)\right)^{\prime} \rightarrow H_{p}^{1}\left(0, T ; \mathbb{R}^{k_{i}}\right)$ given by $J_{i} h=u_{h}\left(h \in\left(H_{p}^{1}\left(0, T ; \mathbb{R}^{k_{i}}\right)\right)^{\prime}\right)$, where $u_{h} \in H_{p}^{1}\left(0, T ; \mathbb{R}^{k_{i}}\right)$ is the weak solution of the problem

$$
\left\{\begin{array}{l}
-u^{\prime \prime}+a_{i}^{2} u=h \quad \text { on }(0, T) \\
u(0)-u(T)=u^{\prime}(0)-u^{\prime}(T)=0
\end{array}\right.
$$

For every $h \in L^{2}\left([0, T] ; \mathbb{R}^{k_{i}}\right)$ we have

$$
\left|J_{i} h\right|_{i}^{2}=\left(J_{i} h, J_{i} h\right)_{i}=\left(h, J_{i} h\right)_{L^{2}} \leq|h|_{L^{2}}\left|J_{i} h\right|_{L^{2}} \leq \frac{1}{a_{i}}|h|_{L^{2}}\left|J_{i} h\right|_{i},
$$

hence

$$
\left|J_{1} h_{1}\right|_{i} \leq \frac{1}{a_{i}}|h|_{L^{2}} .
$$

Associate to the second and the third equation from (3.1) the functionals

$$
E_{2}, E_{3}: H_{p}^{1}\left(0, T ; \mathbb{R}^{k_{1}}\right) \times H_{p}^{1}\left(0, T ; \mathbb{R}^{k_{2}}\right) \times H_{p}^{1}\left(0, T ; \mathbb{R}^{k_{3}}\right) \rightarrow \mathbb{R}
$$

defined by

$$
E_{2}(u, v, w)=\frac{1}{2}|v|_{2}^{2}-\int_{0}^{T} f_{2}(t, u(t), v(t), w(t)) d t
$$

and

$$
E_{3}(u, v, w)=\frac{1}{2}|w|_{3}^{2}-\int_{0}^{T} f_{3}(t, u(t), v(t), w(t)) d t
$$


According to [4, Theorem 1.4] we have

$$
E_{22}(u, v, w)=L_{2} v-\nabla_{y} f_{2}(\cdot, u, v, w),
$$

or equivalently, for any $\varphi \in H_{p}^{1}\left(0, T ; \mathbb{R}^{k_{2}}\right)$,

$$
\begin{aligned}
\left(E_{22}(u, v, w), \varphi\right) & =\left(L_{2} v, \varphi\right)-\left(\nabla_{y} f_{2}\left(u, v, w, u^{\prime}, w^{\prime}\right), \varphi\right) \\
& =\left(v-J_{2} \nabla_{y} f_{2}, \varphi\right)_{2} .
\end{aligned}
$$

Hence $E_{22}(u, v, w)=v-J_{2} \nabla_{y} f_{2}$. Similarly,

$$
E_{33}(u, v, w)=w-J_{3} \nabla_{z} f_{3} .
$$

On the other hand, system (3.1) is equivalent to the following fixed point equation

$$
\left\{\begin{array}{l}
N_{1}(u, v, w)=u \\
N_{2}(u, v, w)=v \\
N_{3}(u, v, w)=w
\end{array}\right.
$$

where

$$
\begin{aligned}
& N_{1}(u, v, w)=J_{1} f_{1}\left(\cdot, u, v, w, u^{\prime}\right), \\
& N_{2}(u, v, w)=J_{2} \nabla_{y} f_{2}(\cdot, u, v, w), \\
& N_{3}(u, v, w)=J_{3} \nabla_{z} f_{3}(\cdot, u, v, w) .
\end{aligned}
$$

Related to $f_{1}, f_{2}, f_{3}$ we assume that the following Lipschitz conditions hold for some constants $a_{i j}$ :

$$
\begin{gathered}
\left|f_{1}\left(t, x_{1}, \ldots, x_{4}\right)-f_{1}\left(t, \overline{x_{1}}, \ldots, \overline{x_{4}}\right)\right| \leq \sum_{j=1}^{4} a_{1 j}\left|x_{j}-\overline{x_{j}}\right| \\
\left|\nabla_{y} f_{2}\left(t, x_{1}, x_{2}, x_{3}\right)-\nabla_{y} f_{2}\left(t, \overline{x_{1}}, \overline{x_{2}}, \overline{x_{3}}\right)\right| \leq \sum_{j=1}^{3} a_{2 j}\left|x_{j}-\overline{x_{j}}\right|, \\
\left|\nabla_{z} f_{3}\left(t, x_{1}, x_{2}, x_{3}\right)-\nabla_{z} f_{3}\left(\overline{x_{1}}, \overline{x_{2}}, \overline{x_{3}}\right)\right| \leq \sum_{j=1}^{3} a_{3 j}\left|x_{j}-\overline{x_{j}}\right| .
\end{gathered}
$$

Then

$$
\begin{aligned}
& \left|N_{1}(u, v, w)-N_{1}(\bar{u}, \bar{v}, \bar{w})\right|_{1}=\left|J_{1}\left(f_{1}\left(\cdot, u, v, w, u^{\prime}\right)-f_{1}\left(\cdot \bar{u}, \bar{v}, \bar{w}, \bar{u}^{\prime}\right)\right)\right|_{1} \\
& \leq \frac{1}{a_{1}}\left|f_{1}\left(\cdot, u, v, w, u^{\prime}\right)-f_{1}\left(\cdot \bar{u}, \bar{v}, \bar{w}, \bar{u}^{\prime}\right)\right|_{L^{2}} \\
& \leq \frac{1}{a_{1}}\left(\int_{0}^{T}\left(a_{11}|u(t)-\bar{u}(t)|+a_{14}\left|u^{\prime}(t)-\bar{u}^{\prime}(t)\right|\right)^{2} d t\right)^{\frac{1}{2}} \\
& +\frac{a_{12}}{a_{1}}|v-\bar{v}|_{L^{2}}+\frac{a_{13}}{a_{1}}|w-\bar{w}|_{L^{2}} \\
& \leq \frac{1}{a_{1}}\left(\left(\frac{a_{11}}{a_{1}}\right)^{2}+a_{14}^{2}\right)^{\frac{1}{2}}|u-\bar{u}|_{1}+\frac{a_{12}}{a_{1}}|v-\bar{v}|_{L^{2}}+\frac{a_{13}}{a_{1}}|w-\bar{w}|_{L^{2}} .
\end{aligned}
$$


Is clear that $|v-\bar{v}|_{L^{2}} \leq \frac{1}{a_{2}}|v-\bar{v}|_{2}$ and $|w-\bar{w}|_{L^{2}} \leq \frac{1}{a_{3}}|w-\bar{w}|_{3}$. Hence, the above inequality becomes

$$
\begin{aligned}
& \left|N_{1}(u, v, w)-N_{1}(\bar{u}, \bar{v}, \bar{w})\right|_{1} \\
\leq & \frac{1}{a_{1}}\left(\left(\frac{a_{11}}{a_{1}}\right)^{2}+a_{14}^{2}\right)^{\frac{1}{2}}|u-\bar{u}|_{1}+\frac{a_{12}}{a_{1} a_{2}}|v-\bar{v}|_{2}+\frac{a_{13}}{a_{1} a_{3}}|w-\bar{w}|_{3} .
\end{aligned}
$$

For $N_{2}(u, v, w)$ we obtain the following estimate

$$
\begin{aligned}
\left|N_{2}(u, v, w)-N_{2}(\bar{u}, \bar{v}, \bar{w})\right|_{2} & \leq\left|J_{2} \nabla_{y} f_{2}(\cdot, u, v, w)-\nabla_{y} f_{2}(\cdot, \bar{u}, \bar{v}, \bar{w})\right|_{2} \\
& \leq \frac{1}{a_{2}}\left|\nabla_{y} f_{2}(\cdot, u, v, w)-\nabla_{y} f_{2}(\cdot, \bar{u}, \bar{v}, \bar{w})\right|_{L^{2}} \\
& \leq \frac{a_{21}}{a_{2}}|u-\bar{u}|_{L^{2}}+\frac{a_{22}}{a_{2}}|v-\bar{v}|_{L^{2}}+\frac{a_{23}}{a_{2}}|w-\bar{w}|_{L^{2}} \\
& \leq \frac{a_{21}}{a_{2} a_{1}}|u-\bar{u}|_{1}+\frac{a_{22}}{a_{2}^{2}}|v-\bar{v}|_{2}+\frac{a_{23}}{a_{2} a_{3}}|w-\bar{w}|_{3} .
\end{aligned}
$$

Similarly

$$
\left|N_{3}(u, v, w)-N_{3}(\bar{u}, \bar{v}, \bar{w})\right|_{3} \leq \frac{a_{31}}{a_{3} a_{1}}|u-\bar{u}|_{1}+\frac{a_{32}}{a_{2} a_{3}}|v-\bar{v}|_{2}+\frac{a_{33}}{a_{3}^{2}}|w-\bar{w}|_{3} .
$$

Therefore, the condition related to $(2.1)$ holds provided that the spectral radius of the matrix

is less than one.

$$
A=\left[\begin{array}{ccc}
\frac{1}{a_{1}}\left(\left(\frac{a_{11}}{a_{1}}\right)^{2}+a_{14}^{2}\right)^{\frac{1}{2}} & \frac{a_{12}}{a_{1} a_{2}} & \frac{a_{13}}{a_{1} a_{3}} \\
\frac{a_{21}}{a_{2} a_{1}} & \frac{a_{22}}{a_{2}^{2}} & \frac{a_{23}}{a_{2} a_{3}} \\
\frac{a_{31}}{a_{3} a_{1}} & \frac{a_{32}}{a_{2} a_{3}} & \frac{a_{33}}{a_{3}^{2}}
\end{array}\right]
$$

In what follows we are trying to establish conditions for $E_{2}(u, \cdot, w)$ and $E_{3}(u, v, \cdot)$ to be bounded from below. To this aim, assume that for $i \in\{2,3\}$ and $j \in\{1,2,3,4\}$, there are $\sigma_{i j} \in L^{1}\left(0, T ; \mathbb{R}_{+}\right)$and $\gamma_{i} \in \mathbb{R}$ with $\gamma_{i}^{2}<\frac{a_{i}^{2}}{2}$ such that

$$
f_{2}(t, x, y, z) \leq \gamma_{2}^{2}|y|^{2}+\sigma_{21}(t)|x|+\sigma_{22}(t)|y|+\sigma_{23}(t)|z|+\sigma_{24}(t)
$$

and

$$
f_{3}(t, x, y, z) \leq \gamma_{3}^{2}|z|^{2}+\sigma_{31}(t)|x|+\sigma_{32}(t)|y|+\sigma_{33}(t)|z|+\sigma_{34}(t) .
$$

Then taking into account the continuous embedding of $H_{p}^{1}\left(0, T ; \mathbb{R}^{k_{i}}\right)$ into $C\left([0, T] ; \mathbb{R}^{k_{i}}\right)$, we obtain

$$
\begin{aligned}
& E_{2}(u, v, w)=\int_{0}^{T}\left(\frac{1}{2}\left|v^{\prime}(t)\right|^{2}+\frac{a_{2}^{2}}{2}\left|v^{2}(t)\right|-f_{2}(t, u(t), v(t), w(t))\right) d t \\
& \geq \int_{0}^{T}\left(\frac{1}{2}\left|v^{\prime}(t)\right|^{2}+\frac{1}{2}\left(a_{2}^{2}-2 \gamma_{2}^{2}\right) v^{2}(t)-\sigma_{21}(t)|u(t)|-\sigma_{22}(t)|v(t)|-\sigma_{23}(t)|w(t)|-\sigma_{24}(t)\right) d t \\
& \geq\left(1-\frac{2 \gamma_{2}^{2}}{a_{2}^{2}}\right)|v|_{2}^{2}-C_{21}|u|_{1}-C_{22}|v|_{2}-C_{23}|w|_{3}-C_{24}
\end{aligned}
$$


for some constants $C_{2 j}, j-1,2,3,4$.

This shows us that $E_{2}(u, v, w) \rightarrow \infty$ as $|v|_{2} \rightarrow \infty$. Similarly, $E_{3}(u, v, w) \rightarrow \infty$ as $|w|_{3} \rightarrow \infty$. Thus the functionals $E_{2}(u, \cdot, w)$ and $E_{3}(u, v, \cdot)$ are coercive. Then, as in [7, Lemma 4.1], these functionals are bounded from bellow.

Finally, assume that for $i \in\{2,3\}$, there are $L^{1}$-Carathéodory functions $g_{i 1}, g_{i 2}:(0, T) \times \mathbb{R}^{k_{i}} \rightarrow \mathbb{R}$ of coercive type such that

$$
g_{21}(t, y) \leq f_{2}(t, x, y, z) \leq g_{22}(t, y)
$$

and

$$
g_{31}(t, z) \leq f_{3}(t, x, y, z) \leq g_{32}(t, z)
$$

for all for all $(x, y, z) \in \mathbb{R}^{k_{1}} \times \mathbb{R}^{k_{2}} \times \mathbb{R}^{k_{3}}$ and $t \in(0, T)$. Here, for example, by the coercivity of $g_{21}(t, y)$ we mean that

$$
\frac{1}{2}|v|_{2}^{2}-\int_{0}^{T} g_{21}(t, v) d t \rightarrow \infty \quad \text { as } \quad|v|_{2} \rightarrow \infty .
$$

Fix $a>0$. Using the above assumption one has

$$
\inf _{v \in H_{p}^{1}} E_{2}(u, \cdot, w)+a \leq \inf _{v \in H_{p}^{1}}\left(\frac{1}{2}|v|_{2}^{2}-\int_{0}^{T} g_{21}(t, v) d t\right)+a .
$$

By the coercivity of $g_{22}$, there exists $R_{2}>0$ such that

$$
\inf _{v \in H_{p}^{1}}\left(\frac{1}{2}|v|_{2}^{2}-\int_{0}^{T} g_{21}(t, v) d t\right)+a \leq \frac{1}{2}|v|_{2}^{2}-\int_{0}^{T} g_{22}(t, v) d t,
$$

for all $|v|_{2} \geq R_{2}$. Now, for $|v|_{2} \geq R_{2}$ and all $(u, w) \in H_{p}^{1}\left(0, T ; \mathbb{R}^{k_{1}}\right) \times H_{p}^{1}\left(0, T ; \mathbb{R}^{k_{3}}\right)$, using again (3.10) we obtain

$$
E_{2}(u, v, w) \geq \frac{1}{2}|v|_{2}^{2}-\int_{0}^{T} g_{22}(t, v) d t \geq \inf _{v \in H_{p}^{1}} E_{2}(u, \cdot, w)+a,
$$

as desired. The similar inequality for $E_{3}$ can be established analogously.

Under the assumptions (3.4), (3.5),(3.6), (3.8), (3.9), (3.10), (3.11) and if the spectral radius of matrix (3.7) is less than one, then all the hypotheses of Theorem 2.1 are fulfilled.

\section{References}

[1] Bełdzinski, M., Galewski, M., Nash-type equilibria for systems of non-potential equations, Appl. Math. Comput., 385(2020), 125456.

[2] Benedetti, I., Cardinali, T., Precup, R., Fixed point-critical point hybrid theorems and applications to systems with partial variational structure, submitted.

[3] Cournot, A., The mathematical principles of the theory of wealth, Economic J.,1838.

[4] Mawhin, J., Willem, M., Critical Point Theory and Hamiltonian Systems, Springer, Berlin, 1989.

[5] Nash, J., Non-cooperative games, Ann. of Math., 54(1951), 286-295.

[6] Precup, R., Methods in Nonlinear Integral Equations, Springer, Amsterdam, 2002. 
[7] Precup, R., Nash-type equilibria and periodic solutions to nonvariational systems, Adv. Nonlinear Anal., 4(2014), 197-207.

\section{Andrei Stan}

Babeş-Bolyai University,

Faculty of Mathematics and Computer Sciences,

1, Kogălniceanu Street,

400084 Cluj-Napoca, Romania

e-mail: stan.andrey@yahoo.com 